\title{
INVESTIGATING THE PROPERTIES OF PLASTIC RESIN PELLETS FOUND IN THE COASTAL AREAS OF LESVOS ISLAND
}

\author{
H.K. KARAPANAGIOTI* \\ I. KLONTZA
}

\author{
Marine Sciences Department \\ University of the Aegean \\ University Hill \\ GR81100 Mytilene, Greece
}

*to whom all correspondence should be addressed:

\begin{abstract}
Plastic pellets (small granules, 1-5 millimeters in diameter) are widely distributed in the ocean and coastal zones all over the world. They are an industrial raw material for the plastic industry and are unintentionally released to the environment both during manufacturing and transport. These pellets are highly persistent and because of their hydrophobic nature they have being identified as carriers of toxic chemicals in the marine environment. Several marine organisms accumulate plastic particles in their stomach. A positive correlation between the mass of ingested plastic and PCBs in fat tissues has been documented. Also, plastic pellets are proposed as indicators of organic pollution in the sea. However, the sorption mechanisms of organic contaminants onto plastic pellets are not yet well understood. For this reason we collected such pellets from various beaches in Lesvos island. Since this material is highly heterogeneous we also obtained, from plastic manufacturers, virgin pellets made from polyethylene and polypropylene. Phenanthrene was used as the model sorbate. It is a compound with high toxicity and abundance in the environment and relatively easy to use in the laboratory. Also, there are many publications dealing with phenanthrene sorption onto different materials and our results are easily compared with previous research. Batch experiments were performed to determine the sorption kinetics of the pellets. Factors under investigation included pellet material, degree of pellet erosion, and sorption kinetic behavior. All of the five beaches in Lesvos island investigated had plastic pellets on the sand surface. All of these plastic pellets were eroded and $2 / 3$ of them had similar appearance to virgin polyethylene pellets obtained from plastic manufacturers. Pellet color is not indicative of erosion that will lead to varying sorption kinetic behavior. Pellet density could not be used to identify pellets sampled from the beach. Apparent sorption coefficient increases with time for all pellets investigated. Thus, sorption into plastic pellets is limited by diffusion in the polymer phase. $2 / 3$ of the plastic eroded pellets demonstrate sorption kinetics similar to virgin polyethylene pellets after 38 days.
\end{abstract}

KEYWORDS: Plastic pellets, coastal zone pollution, polyethylene pellets, polypropylene pellets, diffusion-limited sorption

\section{INTRODUCTION}

Plastics are synthetic organic polymers that have existed for just over a century [1]. The versatility of these materials has lead to a great increase in their use over the past three decades; they have rapidly moved into all aspects of everyday life. Plastic is biodegradation resistant and thus stays in the environment for a long period of time.

Plastic pellets (small granules, 1-5 millimeters in diameter) are raw material used for the production of many different plastic products. They are widely distributed in the ocean all over the world. These plastic pellets can be unintentionally released to the marine environment during manufacturing and transport. Plastic pellets can also be directly introduced to the 
ocean through accidental spills during shipping [2]. They have been found on beaches all over the world and on the sea floor of the oceans. Although these materials are highly heterogeneous the major classes of plastics commonly found in the marine environment are polyethylene, polypropylene, and Nylon 6 or $66[3,4]$. The lifetime of a plastic material in the marine environment is quite variable and depends upon the intensity of the different factors contributing to the breakdown available at the location of interest. It depends for instance, on the temperature of the water column, on the amount of solar UV-B isolation, and the biotic potential of the environment [3].

Several marine organisms accumulate plastic particles in their stomach. A study done on 1033 birds collected of the coast of North Carolina in the USA found that individuals from 55\% of the species recorded had plastic particles in their body [5]. The authors obtained evidence that some seabirds select specific plastic shapes and colors, mistaking them for prey items. The same attitude is also observed in other animals such as fish, turtles, etc when they are searching for food. Planktivores are more likely to confuse plastic pellets with their prey than do piscivores, therefore the former have a higher incidence of ingested plastics $[6,7]$.

Plastic pellets can transfer chemical pollutants to the organisms including polychlorinate biphenyls (PCB's), 1,1- dichloro- 2,2- chlorophenylethylene (DDE) and nonylphenols (NP), which have no natural sources and may have adverse health effects. Such pollutants may absorb into plastic pellets from seawater and show a steady increase in concentration with exposure time [4]. There are two possible mechanisms by which chemicals are incorporated in the marine plastic pellets. The first mechanism is absorption of hydrophobic chemicals onto plastic pellets from seawater because of the low polarity of the plastic surfaces. The other mechanism may be plastic additives and related chemicals contained within the pellet [2]. However, the sorption mechanisms of organic contaminants into plastic pellets are not yet well understood.

The present study has focused on (a) investigating the properties of plastic pellets found on beaches in Lesvos island and (b) compare their sorptive behavior with virgin (polyethylene and polypropylene) pellets. Phenanthrene is used as the model sorbate in batch experiments that are used to determine the sorption kinetics of the pellets. Factors under investigation include pellet material, degree of pellet erosion and sorption kinetic behavior.

\section{MATERIALS AND METHODS}

Plastic pellets were sampled from several coastal zones in Lesvos Island. In these sandy coastal zones, plastic pellets can be easily found on the surface. Samples were taken from the high tide line as well as from the berm or the upper part of the beach. All samples were cleaned from sand and other materials and were visually separated by morphology. A density-based identification method of polymer pellets is used in the present study [8]. Water and $45.5 \%$ by volume isopropyl alcohol solution were used as solutions of known density; 1 and $0.95 \mathrm{~g} \mathrm{ml}^{-1}$, respectively. Virgin pellets made from polyethylene and polypropylene were also obtained from local plastic manufacturers.

Phenanthrene was used as the model chemical in this study. Phenanthrene $\left(\mathrm{C}_{14} \mathrm{H}_{10}\right)$ is a three-ring polycyclic aromatic hydrocarbon with the following properties (a) molecular mass $178 \mathrm{~g} \mathrm{~mol}^{-1}$, (b) solubility $1.29 \mathrm{mg} \mathrm{l}^{-1}$ at $25^{\circ} \mathrm{C}$, and (c) Henry's law constant $2.610^{-5} \mathrm{~atm} \mathrm{~m}^{3} \mathrm{~mol}^{-1}$ [9]. Phenanthrene was chosen because of its moderate to high hydrophobicity (log $\mathrm{K}_{\mathrm{ow}}=4.6 ;[9]$ ), low volatility (Henry's law constant), low biodegradability, and simplicity of analysis. The sorption behavior of phenanthrene with different materials is widely studied and experimental results should be easily comparable to literature results. Phenanthrene was prepared as a $1000 \mathrm{mg} \mathrm{l}^{-1}$ stock solution in methanol.

Test solutions were prepared in synthetic freshwater (water with $0.030 \mathrm{~g} \mathrm{l}^{-1} \mathrm{CaCl}_{2}, 0.018 \mathrm{~g} \mathrm{l}^{-1}$ $\mathrm{CaSO}_{4}, 0.017 \mathrm{~g} \mathrm{l}^{-1} \mathrm{NaHCO}_{3}$ ). Test solutions were also prepared in synthetic seawater (water with $31 \mathrm{~g} \mathrm{I}^{-1} \mathrm{NaCl}, 10 \mathrm{~g} \mathrm{I}^{-1} \mathrm{MgSO}_{4} .7 \mathrm{H}_{2} \mathrm{O}, 0.04 \mathrm{~g} \mathrm{I}^{-1} \mathrm{NaHCO}_{3}$ ). In both cases, sodium azide $\left(\mathrm{NaN}_{3}\right)$ was added at $0.2 \mathrm{~g} \mathrm{l}^{-1}$ to inhibit bacterial growth and thus biodegradation during batch studies.

All sorption kinetic experiments were conducted in triplicate in $120 \mathrm{ml}$ amber glass bottles with Teflon-coated septa. The initial phenanthrene concentration was $100 \mathrm{~g} \mathrm{I}^{-1}$ and one pellet 
$(\sim 0.035 \mathrm{~g})$ was added in each bottle. The bottles were stored at room temperature (around $23^{\circ} \mathrm{C}$ ) in the dark and shaken periodically. Measurements were taken at various time intervals (i.e., 1, 3, 5, 8, 15, 35, 53, 76, 102 days) for both virgin and eroded pellets in synthetic seawater and synthetic freshwater.

Aqueous phenanthrene concentrations were measured by a Perkin-Elmer LS 50B variable wavelength fluorescence detector in cuvette mode. For each batch experiment, triplicate blank samples were prepared and monitored (i.e. phenanthrene without pellets). These blank samples did not indicate any significant phenanthrene degradation or sorptive losses on the glassware for the duration of the experiment.

\section{RESULTS}

\subsection{Occurrence of plastic pellets in Lesvos coastal zones}

Figure 1 shows a map of Lesvos. The black dots on the map show coastal zones where the occurrence of plastic pellets was investigated. In all these five sandy beaches, plastic pellets were found. Sampling took place only on the surface of the sand. Most pellets were found at the top of the beach and less than $5 \%$ found at the splash zone.

\subsection{Classification of Plastic Pellets}

The pellets found on beaches indicated in Figure 1 were classified based on their morphology. All of these plastic pellets were eroded probably by exposure to seawater, sun, and wind. These plastic eroded pellets (PEP) were compared to virgin industrial pellets and were visually classified into polyethylene (PE), polypropylene (PP), and other pellets (Figure 2). A high percentage $(61 \pm 6 \%)$ of polyethylene eroded pellets compared to polypropylene eroded pellets $(21 \pm 6 \%)$ and other pellets $(20 \pm 13 \%)$ were found on the beaches investigated.

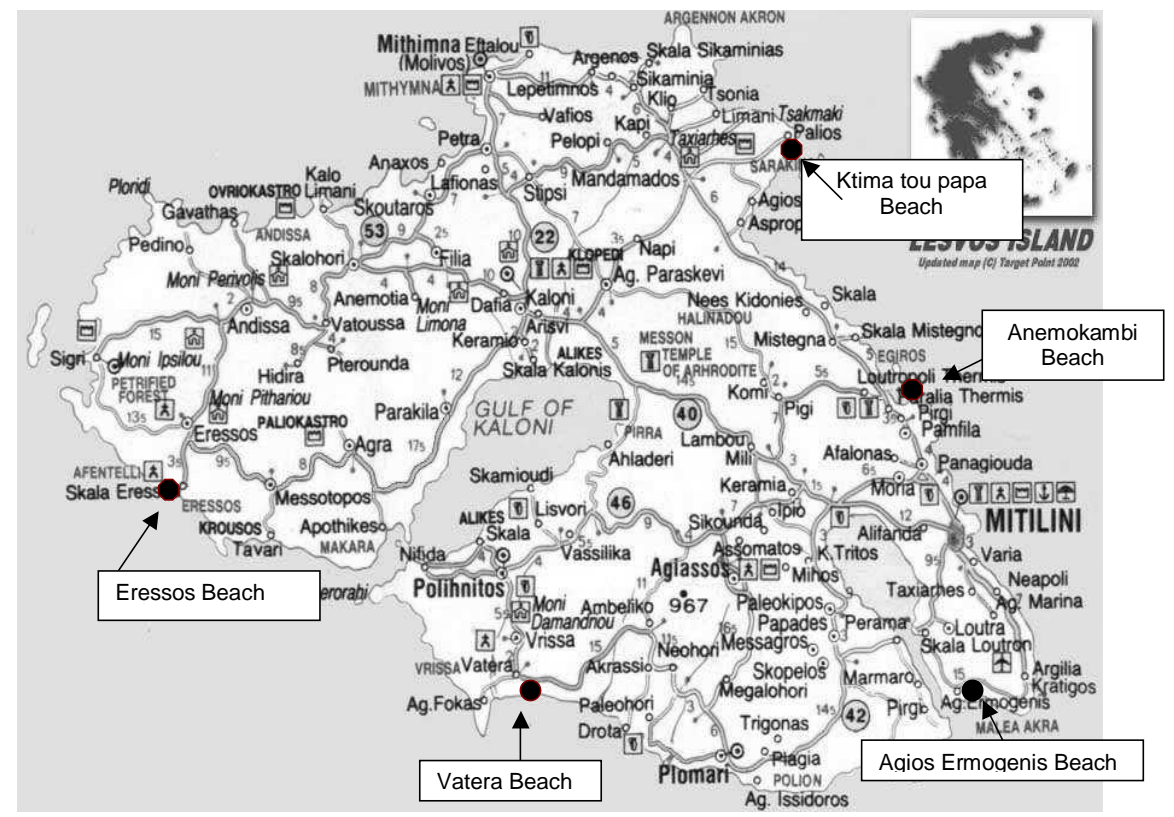

Figure 1. Lesvos Island (black dots: coastal zones where the occurrence of plastic pellets was investigated)

From the PEP found in the examined areas $61 \pm 19 \%$ were white, $20 \pm 12 \%$ were yellow and the other $19 \pm 11 \%$ had orange-brown color (Figure 3 ). PE pellets are normally transparent white and turn white or yellow just from exposure to the sun according to the local plastic supplier. Most of the orange or brown pellets (10\% out of 19\%) collected had a small but visible amount of tar stuck on them. This observation suggests that the orange/brown color could originate from organics transferred from the tar phase into the pellets polymer phase. 


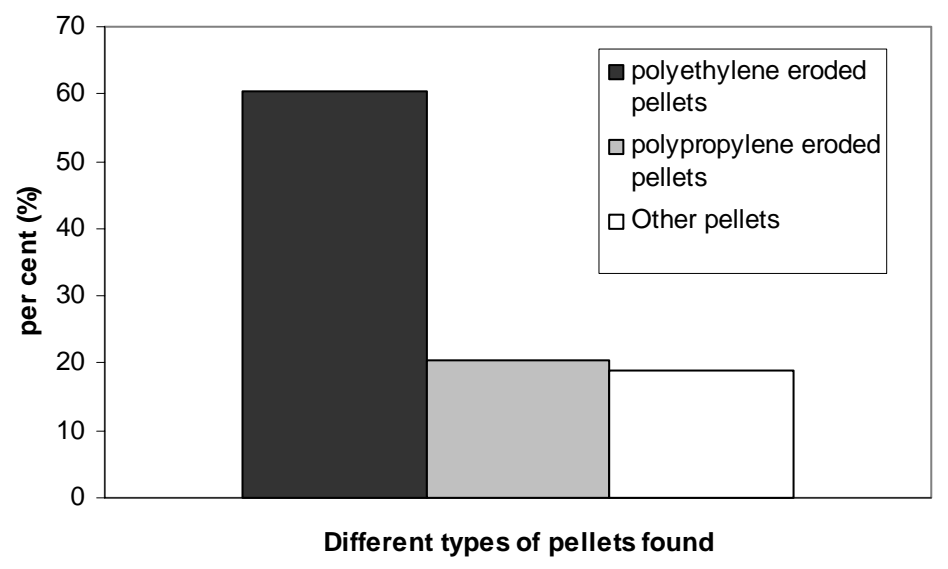

Figure 2. Classification of plastic eroded pellets (PEP) based on their morphology

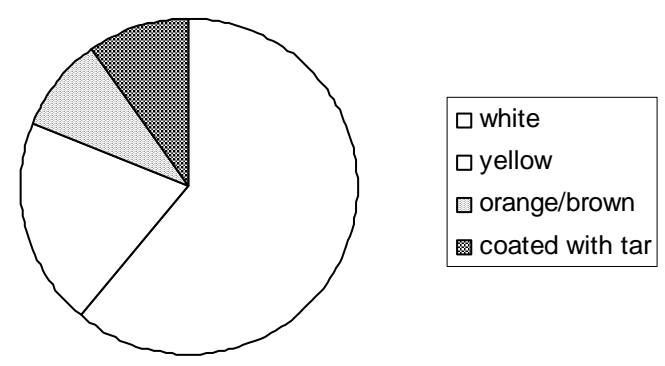

Figure 3. Classification of plastic eroded pellets (PEP) based on their color

All pellets would float in water. Most pellets sink in the isopropyl alcohol solution. Both virgin pellet types (PE and PP) would also sink in the isopropyl alcohol solution although virgin PP is expected to be lighter (0.90-0.91 $\left.\mathrm{g} \mathrm{ml}^{-1}[8]\right)$. This suggests that additives in the manufacturing procedure alter the pellet density and thus this procedure cannot be used to identify unknown material pellets. Nevertheless, there were $9 \pm 8 \%$ pellets that floated in the isopropyl alcohol solution indicating that a pellet material other than PE or PP is found in the coastal areas of Lesvos. The mean weight of PEP collected is $0.035 \pm 0.006 \mathrm{~g}$. The diameter of the pellets ranges from 1 to $5 \mathrm{~mm}$.

\subsection{Sorptive behavior of PEP and comparison to virgin plastic pellets}

Figure 4 presents the kinetics of phenanthrene sorptive uptake by PEP from synthetic fresh water. $K_{\mathrm{da}}$ is the apparent sorption coefficient calculated as the ratio between the chemical sorbed per mass of sorbent $\left(\mu \mathrm{g} \mathrm{Kg}^{-1}\right)$ and the chemical concentration in solution $\left(\mu \mathrm{g} \mathrm{I}^{-1}\right)$. As shown in Figure $4 K_{\text {da }}$ increases with time suggesting a polymer diffusion limited sorption. There is some scattering observed between triplicates and this is due to the heterogeneity of the erosion for the PEP. However, both white and yellow PEP demonstrate similar sorption kinetics. Thus, the pellet color does not represent the degree of erosion on PEP surface.

Figure 5 compares the phenanthrene sorption kinetics of PEP and virgin PE and PP pellets in synthetic seawater. The sorptive uptakes for all three materials are similar on the first day. For PE and PEP sorption continues to increase even after 70 days whereas sorption on PP seems to reach a plateau after 38 days. PP reaches an equilibrium sorption coefficient 1-2 orders of magnitude lower than PE and PEP. 2 out of 3 samples of PEP demonstrate similar sorption kinetics with PE pellets. This is consistent with findings presented in Figure 2 where $61 \%$ of PEP seems to be PE. Pellet surface erosion does not seem to alter sorption kinetics of PEP compared to virgin $P E$ after 38 days. 


\section{CONCLUSIONS}

Plastic pellets were found in different beaches in the island of Lesvos. The color of the pellet cannot be used as indicative of the pellet erosion. Appearance and sorptive behavior indicated that $2 / 3$ of the plastic pellets found on the beaches in this island are made of polyethylene (PE).

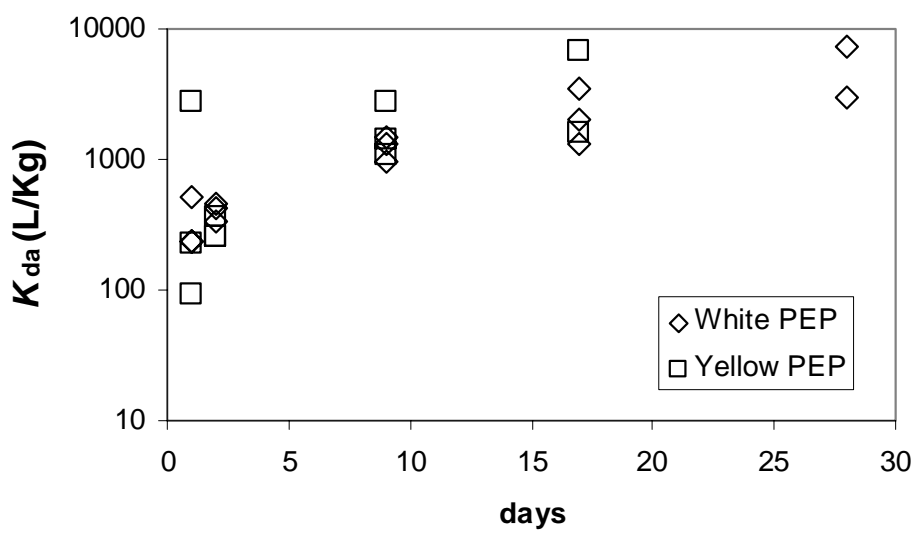

Figure 4. Increase of phenanthrene apparent sorption coefficient $\left(K_{\mathrm{da}}\right)$ with time (PEP: plastic eroded pellets)

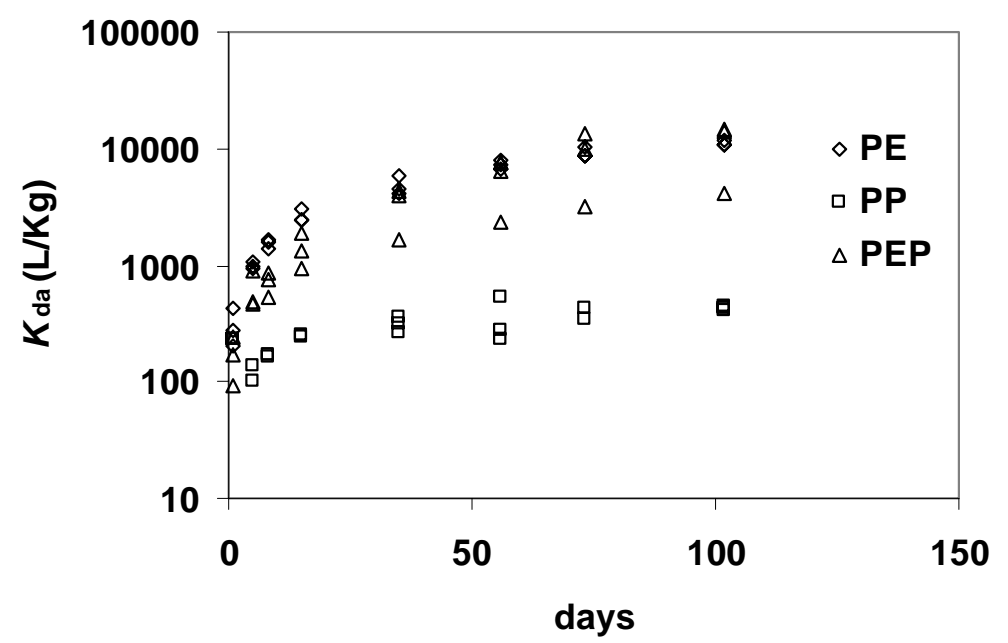

Figure 5. Increase of phenanthrene apparent sorption coefficient $\left(K_{\mathrm{da}}\right)$ with time for different material (PE: polyethylene, PP: polypropylene, PEP: plastic eroded pellets)

\section{REFERENCES}

1. Gorman M., (1993) Environmental Hazards-Marine Pollution, ABC-CLIO Inc,Santa Barbara

2. Mato Y., Isobe T., Takada H., Kanehiro H., Ohtake C., Kaminuma T. (2001) Plastic Resin Pellets as a Transport Medium for Toxic Chemicals in the Marine Environment, Environ.Sci.Technol., 35, 319-324

3. Andrady A.L., (2000) Plastics and their Impacts in the Marine Environment, Proceedings of the International Marine Debris Conference on the Derelict Fishing Gear and the Ocean Environment, Hawai'i Convention Center, Honoloulou, Hawai'i

4. McDermid J.Karla; McMullen L.Tracy. (2004) Quantitative analysis of small plastic debris on beaches in the Hawaiian archipelago, Marine Pollution Bulletin, 48, 790-794

5. Moser M.L. and Lee D.S. (1992) A fourteen year study of plastic ingestion by western North Atlantic seabirds, Colonial Waterbirds, 15, 83-94

6. Azzarello M.Y. and Van Vleet E.S. (1987) Marine Birds and Plastic Pollution, Mar.Ecol.Progr.Ser., 37, 295-303 
7. Derraik G.B. Jose., (2002) The pollution of the marine environment by plastic debris: a review, Marine Pollution Bulletin, 44, 842-852.

8. Katz D.A. (1998) Identification of Polymers http://www.chymist.com/polymeridentification.pdf From original source: Kollman C.S. (1994) Chem 13 News.

9. Montgomery J.H.; Welkom L.M., (1990) Ground Water Chemicals Desk Reference, Lewis Publishers, Inc., Chelsea, Michigan. 